\title{
BMJ Open Not in education, employment, or training (NEET) and risk of alcohol use disorder: a nationwide register-linkage study with 485839 Swedish youths
}

\author{
Helio Manhica (1) , Andreas Lundin, Anna-Karin Danielsson
}

To cite: Manhica H, Lundin A, Danielsson A-K. Not in education, employment, or training (NEET) and risk of alcohol use disorder: a nationwide registerlinkage study with 485839 Swedish youths. BMJ Open 2019;9:e032888. doi:10.1136/ bmjopen-2019-032888

- Prepublication history and additional material for this paper are available online. To view please visit the journal (http:// dx.doi.org/10.1136/bmjopen2019-032888).

Received 11 July 2019 Revised 20 September 2019 Accepted 23 September 2019

A Check for updates

(C) Author(s) (or their employer(s)) 2019. Re-use permitted under CC BY-NC. No commercial re-use. See rights and permissions. Published by BMJ.

Department of Public Health Sciences, Karolinska Institute, Stockholm, Sweden

Correspondence to Dr Helio Manhica; helio.manhica@ki.se

\section{ABSTRACT}

Objective To investigate to what extent being outside education, employment or training after completed secondary education in Sweden might affect the risk of subsequent alcohol use disorders (AUDs), with sociodemographic indicators, such as sex, domicile and origin, taken into account.

Design Population register-based cohort study with 485 839 Swedish youths.

Setting Sweden.

Participants All youths who were born between 1982 and 1991 and were aged between 19 and 24 years when they completed secondary education in Sweden, between 2005 and 2009.

Primary outcome measure Cox regression models were used to estimate the HR of first record of entry into alcohol-related medical care with a diagnosis of an AUD, by level of labour market attachment, from 1 January 2009 to 31 December 2016.

Results About $4 \%$ of the youth population were outside education, employment or training and $25 \%$ were in insecure workforce after they completed secondary education. The risk of AUD was higher among youths in insecure workforce, HR 1.40 (95\% $\mathrm{Cl} 1.30$ to 1.50$)$, and among those outside education, employment or training, HR 1.30 (95\% Cl 1.11 to 1.51), compared with youths within the core workforce, also after adjusting for age, domicile, sex and origin. Being in education was associated with lower HR of AUD, HR=0.84 $(95 \% \mathrm{Cl} 0.78$ to 0.90 )

Conclusion Youths who are in insecure workforce and outside education, employment or training are at higher risk of AUD. Targeted policy actions are needed to support a successful school-work transition to secure equal opportunities for young people.

\section{INTRODUCTION}

The harmful use of alcohol is a major concern for public health. In 2016, alcohol was responsible for about 3 million deaths $(5.3 \%$ of all deaths) globally and $5.1 \%$ of the total global burden of disease, with the prevalence being highest in the WHO European region: $10.1 \%$ of all deaths and $10.8 \%$ of the total burden of disease. ${ }^{1}$

\section{Strengths and limitations of this study}

- The longitudinal study was based on data from a combination of national registers covering the entire youth population living in Sweden.

- The indicator of levels of labour market participation was constructed using information on all possible income sources.

- The small population sizes for specific countries of origin in the migrant population did not allow conducting analyses separated by specific origin.

- Essential confounder variables, which could account for the different patterns of labour market attachment and alcohol-related behaviours, were not available in our data.

In Western countries, a number of studies have shown that alcohol-related mortality and alcohol use disorder (AUD) are more likely to occur among socioeconomically disadvantaged populations. ${ }^{2-4}$ However, this association is not sufficiently understood. While some scholars argue that poor labour market attachment, in terms of unemployment, lower levels of income or low occupational status, is associated with increased risk of excessive alcohol use (social causation), ${ }^{5-7}$ others have suggested the opposite-that is, heavy use of alcohol is a risk factor for poor labour market outcomes (social selection) ${ }^{89}$ In fact, Boden and colleagues ${ }^{10}$ found support for both the social causation and the social selection theory, reporting unemployment to play a causal role in substance misuse (including alcohol), but also the opposite, whereby substance misuse increased the risk of unemployment.

In general, young people are more affected than adults by both labour market disengagement $^{1112}$ and excessive use of alcohol. ${ }^{1}$ Still, previous studies within this field have mainly focused on adult populations. A recent pilot study ${ }^{13}$ found that job security perception 
was associated with depression, anxiety, tobacco smoking and alcohol abuse. However, this study did not examine possible age differences.

Against this background, it is of interest to investigate whether being outside education, employment or training contributes to increased risk of subsequent AUD in youths who are in the process of establishing themselves on the labour market.

\section{Youths and labour market attachment}

The transition from school to successful labour market integration can be a challenge in young people's lives. In fact, the transition often involves moving between different employment statuses, temporary working contracts and other precarious types of employment with low salaries. ${ }^{12}$ These challenges tend to be more pronounced among youths with foreign background than their native peers. Among youth with a migrant background, youth offspring of migrants are less likely to face labour market disadvantages compared with their migrant peers, including youths who arrived as children. This advantage have been explained by a better knowledge of the host country's language, institutional rules and regulations, social networks and the fact that they are less likely to face ethic discrimination. ${ }^{14}$ Thus, in our study, we expect lower rates of labour market attachment among young migrants compared with native-born children of both immigrants and native Swedes.

The high labour market vulnerability facing youths with a migrant background is currently the focus of the policy debate in Sweden and many other European countries, due to the growth of this population caused by a high influx of refugees and asylum seekers. For instance, in 2015, one in five youths aged 15-34 years residing in the European Union (EU) had a migrant background (either being foreign-born or having foreign-born parents). During the same period, about $41 \%$ of all new immigrants to Sweden were youths aged 15-29 years.

The concept NEET-not in education, employment, or training-has been widely used as an indicator for capturing the extent of young people's multifaceted disadvantage in the labour market. It emerged in the UK in the late 1980s, and has been used as an instrument to inform youth-oriented policies in the European Union. ${ }^{15}$ This concept covers all young people who are unemployed and inactive, that is, not enrolled in any formal or non-formal education, as well as those who suffer from long-term sickness or are otherwise unable to work or not available for work. ${ }^{11}$ As in many OECD (Organisation for Economic Co-operation and Development) countries, the rates of NEETs in Sweden are higher among youths with low education (ie, lacking secondary education) than those with tertiary education. ${ }^{16}$ Moreover, youths with a migrant background are overrepresented among NEETs compared with their native peers with comparable education levels. Still, obtaining a secondary education has been found to be protective against the risk of being NEET among all youths, regardless of origin. ${ }^{17}$
Some previous studies have shown that NEET youths are more likely to have poor mental health, including poor self-reported health, substance use (including alcohol) and delinquent behaviours. ${ }^{18-21}$ However, opposing findings, that is, that NEET status does not lead to poor mental health and substance use, have also been reported. ${ }^{22}$ Thus, results from previous studies are inconsistent and have some methodological limitations, such as relying solely on self-reported data, applying cross-sectional designs, with relative small sample sizes, and having unclear durations of the NEET period.

Many prior studies have focused on the association between unemployment and later hospitalisation or death due to alcohol. ${ }^{5623}$ To our knowledge, no study has investigated the association between being outside education, employment or training and AUD in the total youth population of Sweden. It is hypothesised in the current study that AUD would be severely compounded among youth in NEET, as disengagement from the labour market tend to expose youths to a range of negative social and health consequences, including, the harmful use of alcohol.

Therefore, the main objective of this study was to investigate to what extent being NEET in Sweden might affect the risk of subsequent AUD. In addition, we aimed to examine to what extent these possible associations differed with regard to sociodemographic indicators, such as sex, domicile and origin.

\section{METHODS \\ Study population}

The study population comprised all youths who were born between 1982 and 1991 and were alive and residing in Sweden between January 2006 and December 2012, according to the Register of the Swedish Total Population. The dataset allowed us to identify a total of 485839 youths who were aged between 19 and 24 years old when they completed secondary education in Sweden, between 2005 and 2009. Youths with previous AUD and those who did not complete a secondary degree during the follow-up period were excluded from the analyses. The information regarding level of education and year of graduation was obtained from Statistics Sweden's Longitudinal Integration Database for Health Insurance and Labour Market Studies (LISA). The information regarding origin of birth of the study population was obtained from the multi-generation register.

\section{Exposure variable}

We created an indicator of labour market attachment based on information on income sources, for the 3 years consecutively following the year of graduation from secondary school.

\section{Labour market attachment}

The exposure variable was primarily defined based on a model created by Eurostat for estimating the prevalence 
of labour market vulnerability among young people. The model was built using data from the EU Labour Force Survey. It defines NEETs as all youths who remained outside education, employment or training for 6 months or more during the preceding 12 months. In this article, we used an indicator of labour market attachment that has been applied in several studies. ${ }^{1724}$ This indicator was based on information on social assistance, parental leave, disposable income and other sources of income, for the 3 years consecutively following the year for which information on secondary education was retrieved (between 2005 and 2009). Four categories of labour market attachment were conceptualised as follows.

\section{Core workforce}

This category comprises all individuals who can support themselves by means of labour market income. This includes persons with earnings of at least 3.5 price base amounts (PBA) during a year. The PBA is a concept used by the Swedish government to calculate benefits in social insurance programmes. The PBA is calculated based on changes in the general price level, in accordance with the National Insurance Act. This also includes income from social insurance that is linked to employment, such as sickness allowances and payments from the parental insurance system. It excludes income sources such as unemployment benefits, student allowances and disability pensions.

\section{Education}

All persons with annual earnings of $<0.5 \mathrm{PBA}$, or who have been registered as students in any type of education, or have been in some kind of labour market activation programme for at least 100 days, but not in the NEET category.

\section{Insecure workforce}

All persons with a lower attachment to the labour market; with a labour market income of at least 3.5 PBA for no $>1$ year, and $<0.5$ PBA for no $>2$ years. Individuals receiving unemployment insurance for at least two of the 3 years are also included in this category.

\section{Not in education, employment, or training}

All individuals with annual earnings of at most 0.5 PBA, who have received unemployment insurance benefits, incomes from sickness or part-time disability pensions, full disability pension or social assistance.

\section{Outcome}

The outcome variable was retrieved from the Swedish national inpatient and outpatient register. It referred to the first register entry on alcohol-related medical care with a diagnosis of AUD, from 1 January 2009 to 31 December 2016. This included acute intoxication, harmful use, dependence, toxic effects and liver disease (F10:00-F10.99), in accordance with definitions in the tenth edition of the WHO International Classification of Disorders.

\section{Covariates}

We characterised the study population into three categories based on origin, as given in the multi-generation register. Youth migrants were defined as youths born outside Sweden with both parents born abroad. Youth offspring of migrants comprised all Swedish-born youths with at least one parent born abroad. Native Swedish youth comprised all youths born in Sweden with both parents born in Sweden.

Sociodemographic indicators such as age, sex and domicile were retrieved from the LISA register. The variable domicile indicated the place of residence at the beginning of the follow-up period. This was classified into three categories, in accordance with the Swedish Association of Local Authorities and Regions, which is a politically run organisation that represents and advocates for local government in Sweden: big city referred to the metropolitan areas of Sweden's three largest cities: Stockholm, Gothenburg and Malmo. Town covered other predominately urban municipalities in or near medium-sized towns, and rural covered smaller towns/urban areas and rural municipalities. ${ }^{25}$ Age was age in years when secondary education was completed between 2005 and 2009. This ranged from 19 and 24 years. Sex indicated whether the person was female or male.

\section{Statistical analyses}

The analyses were based on person-time measured from January 2009 to whichever of the following that occurred first: death, the first recorded hospital admission due to AUD or the end of the follow-up period on 31 December 2016. In order to minimise possible bias caused by unrecorded migration in our study population, individuals who had a year without any information on household income from work or social benefits were excluded because this was considered to be an indicator of emigration. ${ }^{26}$

We estimated the incidence of the first record of hospital care due to AUD by the degree of labour market attachment, sex, age, domicile and origin during the follow-up period. Results are presented as incidence rate (IR) with $95 \%$ CI. Thereafter, multivariate analyses were done with Cox's regression analysis of person-years, ${ }^{27}$ to estimate the difference in HRs of first record of hospital care due to AUD, between the categories of labour market attachment, that is, core workforce, education, insecure workforce and NEET. In the Cox regression models, the category of 'core workforce' was seen as the reference category. In model 2, we adjusted for sex and age. Domicile was adjusted for in model 3 and, lastly, origin was adjusted for in model 4 . Estimated results are presented as HRs with $95 \%$ CI

We carried out interaction analyses of sex, domicile and origin in relation to the risk of AUD. We found no or non-substantial interaction effects of sex and origin on the outcome. Nevertheless, there was an interaction 


\begin{tabular}{ll}
\hline $\begin{array}{l}\text { Table } 1 \text { Distribution of sociodemographic characteristics in } \\
\text { the study population }\end{array}$ \\
\hline Sociodemographic characteristics & $\mathbf{n}=\mathbf{4 8 5 8 3 9}$ Population \% \\
\hline $\begin{array}{l}\text { Labour market attachment } \\
\text { Core workforce }\end{array}$ & 34.4 \\
\hline Education & 36.6 \\
\hline Insecure workforce & 25.4 \\
\hline NEET & 3.6 \\
\hline Origin & \\
\hline Native Swedish & 77.4 \\
\hline Migrants' offspring & 15.0 \\
\hline Youth migrant & 7.6 \\
\hline Sex & \\
\hline Male & 51.1 \\
\hline Female & 48.9 \\
\hline Age at graduation from secondary & \\
\hline school (years) & \\
\hline 19 & 85.3 \\
\hline 20 & 11.4 \\
\hline 21 & 2.2 \\
\hline 22 & 0.7 \\
\hline Domicile & 0.2 \\
\hline Large cities & 0.1 \\
\hline Medium-sized towns & 34.5 \\
\hline
\end{tabular}

NEET, not in education, employment, or training.

between domicile and AUD; therefore, we have presented the stratified analyses by domicile in online supplementary appendix 1 . All models were tested for proportional hazard assumption using Schoenfeld residuals. ${ }^{27}$ This assumption was not violated. All analyses were made using STATA 15.

\section{Patient and public involvement}

This research was done without patient or public involvement. Neither were involved in the study design or invited to comment on the study design and main results.

\section{Ethic and patient and public involvement}

The datasets are anonymous and the researchers have no access to any personal information that could identify individuals included in the datasets. The Swedish national registers are protected by special legislation, which makes it possible for researchers to collect certain information without personal consent.

\section{RESULTS}

About $4 \%$ of the youth population were NEET and 25\% were in insecure workforce (table 1 ). The majority of youths $(77.3 \%)$ were native Swedish, $15 \%$ were offspring of migrants and $7.6 \%$ born abroad. The majority of the youths lived in medium-sized towns $(42.5 \%)$, while fewest lived in smaller towns/rural areas (23\%). About $96 \%$ of the youth population graduated at the age of 19 or 20 years.

The IR of AUD (online supplementary appendix 1) was higher among youths in insecure work 217.7 (95\% CI 207.1 to 228.7), followed by NEETs 171.8 (95\% CI 148.3 to 198.9$)$, those in the core workforce 155.5 (95\% CI 147.9 to 163.4 ) and in education 127.9 (95\% CI 121.2 to 135.1). In general, AUD rates were higher among males than females. The IRs were higher in migrants' offspring and native Swedes than in youth migrants. AUD increased with age of graduation from secondary education, and were higher among youths living in the metropolitan areas of Sweden's three largest cities, Stockholm, Gothenburg and Malmo (table 2).

The risk of AUD was higher among youths in insecure workforce and NEET compared with youths in the core workforce (table 2). The HRs of AUD was 1.39 (95\% CI 1.20 to 1.50 ) among youths in insecure workforce and 1.28 (95\% CI 1.09 to 1.50 ) among NEET youths, after adjustment for age, sex and domicile. The HR of AUD

Table 2 Cox regression models for first hospital admission/first visit to inpatient/outpatient care due to AUD, by level of employment attachment among youths (male and female) between 2009 and 2016. $n=485839$

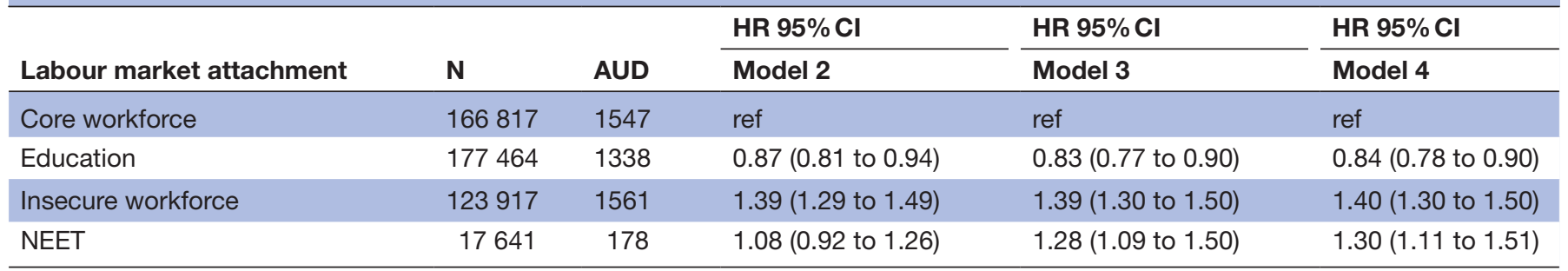

Model 2: adjusted for sex and age.

Model 3: adjusted for sex, age and domicile.

Model 4: adjusted for sex, age, domicile and origin.

$\mathrm{N}$, population.

AUD, alcohol use disorder; NEET, not in education, employment, or training. 
increased slightly to 1.40 (95\% CI 1.30 to 1.50$)$ and 1.30 (95\% CI 1.11 to 1.51 ), respectively, when adjusting for origin. The risk of AUD was lower among youths in education, also after adjustments for the sociodemographic indicators.

Stratified analyses by domicile (online supplementary appendix 2) suggested that, regardless of the characteristics of domicile, the HR of AUD was higher among youths in insecure workforce when compared with those of the core workforce. NEET youths were more likely to have higher risk of AUD if they resided in medium-sized towns.

\section{DISCUSSION}

Our register-based follow-up study suggested that there was a positive and significant association between being in insecure workforce or outside education, employment or training (NEET), and subsequent AUD. In contrast, being in education was associated with lower risk of AUD.

Across the OECD countries, youths face greater challenges in the labour market compared with adults. ${ }^{15}$ Youths often experience multiple periods of unemployment and/or inactivity or move between different employment statuses, with part-time working contracts, temporary contracts and low salaries. ${ }^{12} 15$ The disadvantage has for instance been explained by the fact that youths lack working experience and skills, and, as a consequence, employers lack willingness to hire them. ${ }^{12}{ }^{16}$ In this study, we found that about $4 \%$ of youths were NEET and 25\% were in insecure workforce. This suggests that some youths, despite having graduated from secondary education, may have problems in transiting from school to further education and the core workforce.

Our findings showed that compared with the core workforce, youths in insecure workforce and NEETs have higher risk of AUD. These findings are in line with previous studies showing that labour market disengagement might increase psychological distress and poor mental health. ${ }^{28}{ }^{29}$ These psychological responses, in turn, could lead to excessive use of alcohol for, for example, self-medication or as a coping strategy to deal with feelings of distress. ${ }^{30}$ Our results underscore the effects of job insecurity and involuntary disengagement from the labour market on psychological health and health risk behaviours. ${ }^{31} 32$

The fact that the risk was somewhat higher in youths living in medium-sized towns, and smaller towns/rural areas, than in those in larger cities, calls for further investigations. In fact, this study could not confirm the pattern found in other studies that living in smaller communities was associated with a lower proportion of heavy alcohol consumption and alcohol-related problems. ${ }^{33}$ One potential explanation for the geographical differences could be differences in local labour market (eg, local youth labour market programmes) and selection into labour market participation.

\section{Strengths and limitations}

A major strength of this longitudinal study was that it was based on data from a combination of national registers covering the entire youth population living in Sweden. We were, therefore, able to study a national cohort of youths after they had completed secondary education in Sweden and were transiting from school to labour market. Furthermore, we were able to create an indicator of levels of labour market participation, using information on all possible income sources. This study uses a definition of NEET in line with that proposed by Eurostat in 2016, ${ }^{11}$ although with a longer reference period. We used three consecutive years, capturing those who experienced longterm disadvantages. A further strength of this study is that we excluded all individuals who had a diagnosis of AUD before the beginning of the follow-up period, thus reducing the risk of reverse causality.

This study also had some limitations. First, because of the small population sizes for specific countries of origin in the migrant population, it was not possible to split this category into smaller units and conduct analyses separated by more specific origin. Second, we did not consider information about other possible psychiatric health problems. Therefore, caution must be used in drawing definitive conclusions about the association between labour market engagement and AUD. Third, essential confounder variables, such as cultural values, social support and experiencing discrimination, which could account for the different patterns of labour market attachment and alcohol-related behaviours, were not available in our data. Fourth, being in NEET and having an AUD might share many overlapping risk factors (school, family, friends and so on), on which we had no information in this study. Hence, we are not able to explain the mechanisms that put an individual at greater risk of being in NEET. Our main focus, however, was on the possible consequences rather than the causes. Fifth, caution needs to be taken in how these findings are interpreted because the outcome variable, hospital record due to alcohol-related disorders, implies serious problems related to alcohol misuse. If anything, however, this may have led to an underestimation of the actual problem.

To summarise, compared with youths in the core workforce, youths who are in insecure workforce and outside education, employment or training were at higher risk of AUD. In contrast, youths in education had lower risk of AUD. Further studies are needed to explore the mechanisms underlying the associations between labour market disadvantages and AUD.

Contributors $\mathrm{HM}, \mathrm{AL}$ and $\mathrm{A}-\mathrm{KD}$ contributed to the study design. HM carried out the statistical analyses (supported by AL), drafted the initial manuscript, reviewed and revised the manuscript. HM and A-KD conceptualised and drafted the initial manuscript. AL and A-KD revised the draft of the manuscript as well as the interpretation of the results. All authors approved the final manuscript as submitted and agree to be accountable for all aspects of the work.

Funding This study was supported by grants provided by the Research Council for Health Working Life and Welfare (Forte: 2016-07108).

Competing interests None declared. 
Patient consent for publication Not required.

Ethics approval The Regional Ethics Committee in Stockholm approved the study before any records were linked (decision number: 2016/987-32).

Provenance and peer review Not commissioned; externally peer reviewed.

Data availability statement № data are available.

Open access This is an open access article distributed in accordance with the Creative Commons Attribution Non Commercial (CC BY-NC 4.0) license, which permits others to distribute, remix, adapt, build upon this work non-commercially, and license their derivative works on different terms, provided the original work is properly cited, appropriate credit is given, any changes made indicated, and the use is non-commercial. See: http://creativecommons.org/licenses/by-nc/4.0/.

ORCID iD

Helio Manhica http://orcid.org/0000-0003-3893-2543

\section{REFERENCES}

1 WHO. Global status report on alcohol and health 2018. Geneva World Health Organization; 2018.

2 Katikireddi SV, Whitley E, Lewsey J, et al. Socioeconomic status as an effect modifier of alcohol consumption and harm: analysis of linked cohort data. Lancet Public Health 2017;2:e267-76.

3 Budhiraja M, Landberg J. Socioeconomic disparities in alcoholrelated mortality in Sweden, 1991-2006: a register-based follow-up study. Alcohol Alcohol 2016;51:307-14

4 Erskine S, Maheswaran R, Pearson T, et al. Socioeconomic deprivation, urban-rural location and alcohol-related mortality in England and Wales. BMC Public Health 2010;10:99.

5 Backhans MC, Balliu N, Lundin A, et al. Unemployment is a risk factor for hospitalization due to alcohol problems: a longitudinal study based on the Stockholm public health cohort (SPHC). J Stud Alcohol Drugs 2016;77:936-42.

6 Lundin A, Backhans M, Hemmingsson T. Unemployment and hospitalization owing to an alcohol-related diagnosis among middleaged men in Sweden. Alcohol Clin Exp Res 2012;36:663-9.

7 Popovici I, French MT. Does unemployment lead to greater alcohol consumption? Ind Relat 2013;52:444-66.

8 Johansson E, Alho H, Kiiskinen $\mathrm{U}$, et al. The association of alcohol dependency with employment probability: evidence from the population survey 'Health 2000 in Finland'. Health Econ 2007;16:739-54

9 MacDonald Z, Shields MA. Does problem drinking affect employment? Evidence from England. Health Econ 2004;13:139-55.

10 Boden JM, Lee JO, Horwood LJ, et al. Modelling possible causality in the associations between unemployment, cannabis use, and alcohol misuse. Soc Sci Med 2017;175:127-34.

11 Eurofound. Exploring the diversity of NEETs. Luxembourg: Publications Office of the European Union, 2016.

12 Quintini G, Martin JP, Martin S. The Changing Nature of the Schoolto-Working Transition Process in OECD Contries(2007). WDA-HSG Discussion Paper No 2007.

13 Bert F, Gualano MR, Thomas R, et al. Exploring the possible health consequences of job insecurity: a pilot study among young workers. Gaceta Sanitaria 2018.

14 OECD/EU. Indicators of immigrant integration 2015: settling in. Paris/ EU, Brussels: OECD Publishing, 2015. https://doi.org/
15 Eurofound. NEETs: young people not in employment, education or training: characteristics, costs and policy responses in Europe. Luxembourg: Publications Office of the European Union, 2012.

16 OECD. Investing in youth: Sweden. Paris: OECD Publishing, 2016. https://doi.org/

17 Manhica H, Berg L, Almquist YB, et al. Labour market participation among young refugees in Sweden and the potential of education: a national cohort study. Journal of Youth Studies 2018:1-8.

18 O'Dea B, Glozier N, Purcell R, et al. A cross-sectional exploration of the clinical characteristics of disengaged (NEET) young people in primary mental healthcare. BMJ Open 2014;4:e006378.

19 Henderson JL, Hawke LD, Chaim G. Not in employment, education or training: mental health, substance use, and disengagement in a multi-sectoral sample of service-seeking Canadian youth. Child Youth Serv Rev 2017;75:138-45.

20 Stewart $\mathrm{CH}$, Berry P, Przulj D, et al. Cancer-Related health behaviours of young people not in education, employment or training ('NEET'): a cross-sectional study. BMC Cancer 2017; $17: 165$

21 Benjet C, Hernández-Montoya D, Borges G, et al. Youth who neither study nor work: mental health, education and employment. Salud Pública de México 2012;54:410-7.

22 Baggio S, Iglesias K, Deline S, et al. Not in education, employment, or training status among young Swiss men. longitudinal associations with mental health and substance use. J Adolesc Health 2015;56:238-43.

23 Just-Østergaard E, Mortensen EL, Flensborg-Madsen T. Major life events and risk of alcohol use disorders: a prospective cohort study. Addiction 2018;113:25-33.

24 Bäckman O, Nilsson A. Long-Term consequences of being not in employment, education or training as a young adult. stability and change in three Swedish birth cohorts. European Societies 2016;18:136-57.

25 SKL. The Swedish association of local authorities and regions (salar), 2019. Available: https://skl.se/tjanster/englishpages.411.html [Accessed 20 May 2019].

26 Weitoft GR, Gullberg A, Hjern A, et al. Mortality statistics in immigrant research: method for adjusting underestimation of mortality. Int $J$ Epidemiol 1999;28:756-63.

27 Hosmer Jr DW, Lemeshow S, May S. Applied survival analysis: regression modeling of time-to-event data. Wiley-Interscience, 2008.

28 Meltzer H, Bebbington P, Brugha T, et al. Job insecurity, socio-economic circumstances and depression. Psychol Med 2010;40:1401-7.

29 Kim TJ, von dem Knesebeck O. Perceived job insecurity, unemployment and depressive symptoms: a systematic review and meta-analysis of prospective observational studies. Int Arch Occup Environ Health 2016;89:561-73.

30 Bolton JM, Robinson J, Sareen J. Self-Medication of mood disorders with alcohol and drugs in the National epidemiologic survey on alcohol and related conditions. J Affect Disord 2009;115:367-75.

31 Ashford SJ, Lee C, Content BP. Cause, and consequences of job insecurity: a theory-based measure and substantive test. Academy of Management journal 1989;32:803-29.

32 Coles B, Hutton S, Bradshaw J, et al. Literature review of the costs of being" not in education, employment or training" at age 16-18. 347. Social Policy Research Unit, University of York, 2002.

33 Bränström R, Andréasson S. Regional differences in alcohol consumption, alcohol addiction and drug use among Swedish adults. Scand J Public Health 2008;36:493-503. 